\section{Path-length-resolved optical Doppler perfusion monitoring}

\author{
Babu Varghese, ${ }^{\mathrm{a},}{ }^{*}$ Vinayakrishnan Rajan, ${ }^{\mathrm{a}}$ \\ Ton G Van Leeuwen, ${ }^{a, b}$ and Wiendelt Steenbergen ${ }^{a}$ \\ a University of Twente, Institute for Biomedical Technology \\ BMTI, Biophysical Engineering Group, Faculty of \\ Science and Technology, P.O. Box 217, 7500 AE Enschede, \\ The Netherlands \\ ${ }^{b}$ Academic Medical Center, Laser Center, Meibergdreef 9, \\ 1105 AZ, Amsterdam, The Netherlands
}

\begin{abstract}
We report the first path-length-resolved perfusion measurements on human skin measured with a phase-modulated low-coherence Mach-Zehnder interferometer with spatially separated fibers for illumination and detection. Optical path lengths of Doppler shifted and unshifted light and path-length-dependent Doppler broadening of multiply scattered light from skin are measured from the Doppler broadened interference peaks appearing in the power spectrum. Perfusion and its variations during occlusion are measured in real time for a given optical path length, and the results are compared with the perfusion signal obtained with a conventional laser Doppler perfusion monitor. $\odot 2007$ Society of Photo-Optical Instrumentation Engineers. [DOI: 10.1117/1.2823141]
\end{abstract}

Keywords: Doppler; perfusion; optical path length; interferometry.

Paper 07343LR received Aug. 22, 2007; revised manuscript received Oct. 17, 2007; accepted for publication Oct. 23, 2007; published online Dec. 28, 2007.

The poorly established and biologically uncertain scattering and absorption properties of tissue often complicate noninvasive optical diagnosis in turbid tissues. For instance, in laser Doppler perfusion monitor (LDPM), the mean number of interactions with red blood cells depends on the paths traversed by the detected photons as they penetrate the tissue, and perfusion values averaged over different and basically unknown path lengths are measured. ${ }^{1}$ The depth of light penetration in the tissue depends on the wavelength of the light source, the distance between the transmitting and receiving fibers, and the optical properties of the tissue. This dependence leads to uncertainties in interpreting the Doppler shifted and nonshifted fraction of photons and also in discriminating the fraction of light scattered from superficial (nutrient) and deeper (thermoregulatory) layers of skin. As such, depthresolved perfusion information could be obtained by multiwavelength systems, ${ }^{2-4}$ by varying the distance between transmitting and receiving fibers, ${ }^{5}$ or by using a multichannel laser Doppler probe. ${ }^{6}$ A multiwavelength laser system relies on short wavelength lasers, which are bulky, expensive, and difficult to stabilize. ${ }^{4}$ An alternate approach to depth discrimination based on coherence modulation of semiconductor lasers was presented using the variable coherence properties of the semiconductor laser. ${ }^{7}$ In this technique, they exploited dramatic variations in effective coherence length properties of

\footnotetext{
*Tel: 31534891080; E-mail: b.varghese@utwente.nl
}

certain types of laser diodes by simply regulating the input drive current to these devices. For a long coherence length, all photons interfere, while for a short coherence length only photons with almost the same path length will interfere. This will relatively suppress the deep photons, since the (few) deep photons will only interfere with the few deep photons but not with the (many) shallow photons. However, these methods still give no control over the optical path length traveled by the detected light.

Detection of multiple scattered light and its Doppler spectra as a function of path length may result in more quantitative and reliable tissue structural and functional information. Pathlength-resolved photon intensity, and the associated Doppler spectra on completely dynamic media, can be measured using low coherence interferometry. ${ }^{8-11}$ Path length distributions in almost static and mixed static-dynamic media can be measured by modulating the optical path length in the reference arm. ${ }^{12}$ This will enable path-length-resolved measurements in mixed media such as tissue perfused with blood. Furthermore, we validated the optical path length distributions and pathlength-dependent diffusion broadening of multiple scattered light with Monte Carlo simulations, and diffusive wave spectroscopy, respectively. ${ }^{13}$

In this study, we show the first results on path-lengthresolved laser Doppler perfusion measurements in skin. For a given optical path length, perfusion was measured in real time before, during, and after an occlusion of the upper arm. In all measurements, perfusion was measured simultaneously with a conventional LDPM.

We use a fiber optic Mach-Zehnder interferometer with a superluminescent diode (Inject LM2-850, $\lambda=832 \mathrm{~nm}$, $\Delta \lambda_{\mathrm{FWHM}}=17 \mathrm{~nm}$, coherence length $L_{C}=18 \mu \mathrm{m}$ ) that yields $2 \mathrm{~mW}$ of power from the single-mode pigtail fiber as the light source. We use a single-mode fiber (mode field diameter $=5.3 \mu \mathrm{m}, \mathrm{NA}=0.14$ ) for illumination and multimode graded index fiber (core diameter $=100 \mu \mathrm{m}, \mathrm{NA}=0.29$ ) for detection with a center-to-center separation of $300 \mu \mathrm{m}$. The use of multimode fiber provides a large detection window with a small modal dispersion. The reference beam is polarized using a linear polarizer and the phase is sinusoidally modulated at $6 \mathrm{kHz}$ using an electro-optic broadband phase modulator (New Focus Model 4002) with a peak optical phase shift of 2.04 radians applied to the modulator, so that the power spectrum contains interference peaks at both the phase modulation frequency and higher harmonics. The AC photocurrent is measured with a 12-bit analog-to-digital converter (National Instruments), sampling at $40 \mathrm{kHz}$, averaged over $1000 \mathrm{spec}-$ tra, and was measured in steps of $200 \mu \mathrm{m}$ in air. ${ }^{12-14}$

The fundamental output quantity of a LDPM is the first moment of the power spectrum $P(\omega)$ of the detector signal; in general, the $i$ 'th moment is being defined as

$$
M_{i}=\int_{a}^{b} P(\omega) \omega^{i} d \omega .
$$

Here $a$ and $b$ are device dependent low and high cut-off frequencies. With $i=0$, a quantity is obtained that is proportional to the concentration of moving red blood cells, while $i=1$ describes red blood cell flux, which is the product of concen-

1083-3668/2007/12(6)/060508/3/\$25.00 @ 2007 SPIE 


\section{JBO LETTERS}

tration and the root mean square of the red cell speed, at least for low blood concentrations. ${ }^{15}$ The conventional LDPM (PF5000, Perimed AB, Sweden) uses a laser diode (780 nm) as the light source, a bandwidth of $20 \mathrm{~Hz}$ to $13 \mathrm{kHz}$ [ $a$ and $b$ in Eq. (1)], and a time constant of $0.2 \mathrm{~s}$ was used for monitoring. The fiber optic probe (Probe 408, standard probe) consists of two spatially separated fibers (core diameter $=125 \mu \mathrm{m}, \mathrm{NA}=0.37$ ) with a center-to-center separation of $250 \mu \mathrm{m}$.

Optical path length distributions are obtained by adding the areas of all interference peaks (after subtraction of the background noise, and within a bandwidth of $\pm 2 \mathrm{kHz}$ around all center frequencies) in the power spectrum. The area of the Doppler broadened peak, excluding the statically scattered light contribution at the interference peaks, forms an estimation of the amount of Doppler shifted light at that specific optical path length. The average Doppler shift corresponding to the Doppler shifted light is calculated from the weighted first moments $\left(M_{1} / M_{0}\right)$ of the heterodyne peak at the modulation frequency, after correction for the homodyne signal from the sample arm (skin) and for the reference arm noise [in a bandwidth of $50 \mathrm{~Hz}$ to $2 \mathrm{kHz}$ close to the phase modulation frequency and its higher harmonics, indicated by $a$ and $b$ in Eq. (2)].

$$
M_{i}=\sum_{j=1}^{3} \int_{j \omega_{m}+a}^{j \omega_{m}+b} P(\omega)\left(\omega-j \omega_{m}\right)^{i} d \omega .
$$

Path length distribution measurements were performed on the skin of the dorsal side of the right forearm of a darkskinned healthy human volunteer in the sitting position. The fiber optic probe was inserted into a probe holder ( $\mathrm{PH} \mathrm{08)} \mathrm{that}$ was attached to the skin with a double-sided adhesive tape. The subject rested approximately $10 \mathrm{~min}$ prior to the measurements. For monitoring the perfusion changes in real time, the signal was sampled at $40 \mathrm{kHz}$ for $2.6 \mathrm{~s}$ to get an average of 100 spectra. Simultaneous measurement of perfusion was performed with the PF5000 at a position approximately $3 \mathrm{~cm}$ distant to the probe of the low coherence interferometer. The perfusion was recorded for $30 \mathrm{~s}$ before occlusion, $90 \mathrm{~s}$ during occlusion, $105 \mathrm{~s}$ between occlusions, a second occlusion for another $90 \mathrm{~s}$, and final measurements for $30 \mathrm{~s}$.

We observed that during the arterial occlusion, the broadening of the interference peaks resulting from the Doppler shift imparted by the moving red blood cells to the multiply scattered photons is reduced, whereas the area of the peaks, which represents the intensity of detected photons within a certain optical path length, does not change. The intensity of Doppler shifted and nonshifted photons measured in skin as a function of optical path length are shown in Fig. 1. The fraction of Doppler shifted photons and nonshifted photons averaged over the entire optical path length measured from the respective areas of the optical path lengths are 22 and $78 \%$, respectively. As shown in Fig. 1, the weighted first moment $M_{1} / M_{0}$ of the Doppler shifted light, which represents the average Doppler shift, increased with the optical path length due to the greater probability of interaction of photons with moving scatterers for large optical path lengths.

Figure 2 shows the perfusion signal measured in skin in real time for an optical path length difference of $1.7 \mathrm{~mm}$ in air. The occlusion results in the suppression of mean flow

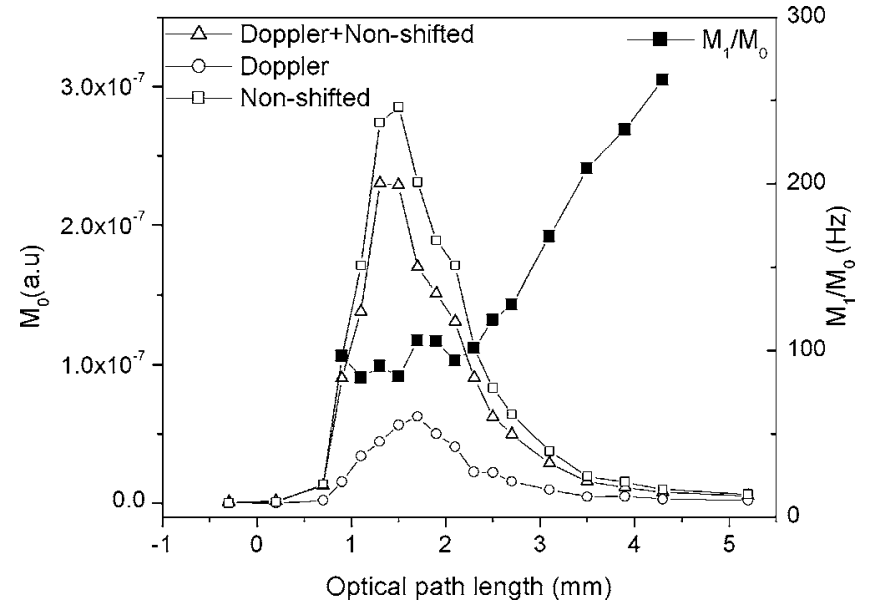

Fig. 1 Intensity of Doppler-shifted, nonshifted photons and the average Doppler shift as a function of optical path length measured in skin.

velocity of red blood cells to nearly zero values (biological zero). The flux of red blood cells (Fig. 2, left, bottom) and the Doppler-shifted fraction of photons are reduced due to occlusion (Fig. 2, left, top). Since the realization of occlusion takes some time, the perfusion decreases gradually and finally drops to biological zero values. After the occlusion is released, the perfusion signal increases above the normal value, an effect called postocclusive reactive hyperemia (PORH). PF5000 traces recorded with a time constant of $0.2 \mathrm{~s}$ are averaged for the same measurement time used in our measurements (2.6 s). Real-time measurements performed with our setup show similar trends as the perfusion readings measured with the PF5000. However, smoother perfusion signals are shown from the PF5000 compared to the fluctuating perfusion signal obtained with our LCI set up. Although the mean depth is of the order of $1 \mathrm{~mm}$, in PF5000, perfusion values averaged over all optical path lengths are measured, which often spans a range of zero to $5 \mathrm{~mm}$. In our setup, the coherence length of the light source acts as a bandpass filter in selecting the photons that have traveled a specific optical path length (for in-

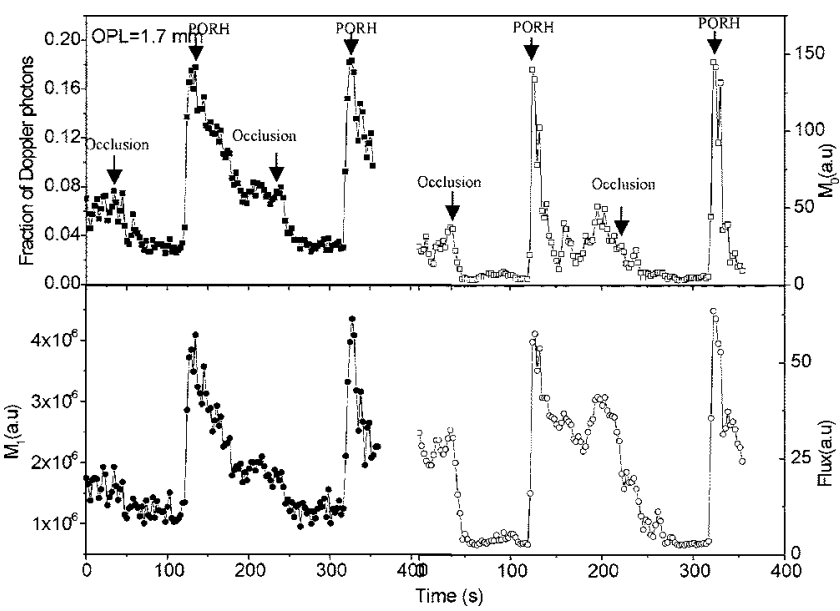

Fig. 2 Real-time monitoring of perfusion measured with our setup (left) for an optical path length of $1.7 \mathrm{~mm}$ and simultaneous measurements with PF5000 (right). 
stance, $1.7 \mathrm{~mm}$ ) in the skin with a tolerance of $\pm 50 \mu \mathrm{m}$, as defined by the path length resolution of the setup. Hence, in a LDPM, due to the large coherence length of the light source, more photons are involved in the interference process, leading to a large signal-to-noise ratio of the raw signal, and a more stable estimation of its spectral moments. The lowest value recorded with PF5000 is relatively lower during occlusion, and the PORH peak with LDPM is shorter in time than with LCI. These variations could result from the fact that both techniques probe different positions (spatial separation of $3 \mathrm{~cm}$ ) and our probe measures physiological perfusion in the superficial layers (with an optical path length of $1.7 \mathrm{~mm}$ ) compared to the perfusion averaged over all path lengths and depths. Also, we only have heterodyne detection, while in LDPM the signal power spectrum is composed of mutual interference of the Doppler-shifted and nonshifted light detected from the tissue. Temporal resolution of $2.6 \mathrm{~s}$ makes it difficult to follow all physiologic changes, such as cardiac cycle and reactive hyperaemia responses. During occlusion, the observed discrepancy between the calibrated zero of the instrument (PF5000) and the "biological zero" level is attributed to the fact that though the blood perfusion is arrested by the inflated cuff, minor Doppler components are recorded by the instrument due to the no-flow laser Doppler signal from vasomotion, Brownian motion from within the vascular compartment and of macromolecules in the inter- and intracellular fluids, and the effects of cuff compression. ${ }^{16}$ The zero on the vertical scale (Fig. 2, left) represents the calibrated instrumental zero of our instrument. The offset between the perfusion readings during occlusion and the calibrated zero of LCI represents the perfusion signal corresponding to the biological zero for an optical path length difference of $1.7 \mathrm{~mm}$. The lowest value recorded with LCI during occlusion is relatively higher compared to PF5000. With an optical path length difference of $1.7 \mathrm{~mm}$, perfusion from superficial layers is only measured. Due to the smaller and more shallow measurement volume in LCI for an OPL of $1.7 \mathrm{~mm}$, the maximum perfusion within the probed volume is smaller, since fewer vessels are probed. Hence, the maximum perfusion value is relatively lower, which makes the minimum perfusion value during occlusion relatively larger.

In this work, we have presented the first path-lengthresolved Doppler measurements of multiply scattered light from skin, using a phase-modulated low coherence MachZehnder interferometer with spatially separated fibers for illumination and detection. Optical path length distributions of multiply scattered light, spanning a range of 0 to $5 \mathrm{~mm}$, have been measured. The path-length-resolved perfusion measured in real time with our setup, for an optical path length of $1.7 \mathrm{~mm}$, and its variations during artery occlusion, showed correlation with the perfusion signal measured using a con- ventional LDPM. The Doppler-shifted fraction of photons, which is related to the blood volume and the flux of red blood cells measured from the first moment, are decreased during occlusion. In general, the path-length-resolved perfusion measurements presented here will overcome the influence of photon path lengths on the measured perfusion signal, and make it possible to perform depth-resolved perfusion measurements. Also, our method allows us to discriminate between the Doppler-shifted and nonshifted fraction of photons in the detected photodetector signal. Further in vivo studies were performed to measure the variations in perfusion to external stimuli, inter-and intraindividual variations in optical path lengths and path-length-resolved Doppler shifts, and to compare these results with the perfusion signal measured with a conventional LDPM. These results will be reported separately in the near future.

\section{Acknowledgments}

This work was sponsored by the Netherlands Technology Foundation STW (Grant TTF 5840) and the Institute for Biomedical Technology of the University of Twente. Perimed AB (Stockholm, Sweden) is acknowledged for providing instrumentation.

\section{References}

1. A. P. Shepherd and P. Å. Öberg, Laser-Doppler Blood Flowmetry, Kluwer Academic, Boston (1990).

2. A. N. Obeid, D. M. Boggett, N. J. Barnett, G. Dougherty, and P. Rolfe, Med. Biol. Eng. Comput. 26, 415-419 (1988).

3. R. J. Gush and T. A. King, Med. Biol. Eng. Comput. 29, 387-392 (1991).

4. L. Duteil, J. C. Bernengo, and W. Schalla, IEEE Trans. Biomed. Eng. BME32, 439-447 (1985).

5. M. Larsson, W. Steenbergen, and T. Strömberg, J. Biomed. Opt. 7(2), 236-243 (2001).

6. A. Liebert, M. Leahy, and R. Maniewski, Med. Biol. Eng. Comput. 36, 740-747 (1998).

7. G. Dougherty, Rev. Sci. Instrum. 63, 3220-3221 (1992).

8. A. Wax, C. Yang, R. R. Dasari, and M. S. Feld, Appl. Opt. 40, 4222-4227 (2001).

9. K. K. Bizheva, A. M. Siegel, and D. A. Boas, Phys. Rev. E 58, 7664-7667 (1998)

10. A. L. Petoukhova, W. Steenbergen, and F. F. M. de Mul, Opt. Lett. 26, 1492-1494 (2001).

11. D. Huang, E. A. Swanson, C. P. Lin, J. S. Schuman, W. G. Stinson, W. Chang, M. R. Hee, T. Flotte, K. Gregory, C. A. Puliafito, and J. G. Fujimoto, Science 254, 1178-1181 (1991).

12. B. Varghese, V. Rajan, T. G. Van Leeuwen, and W. Steenbergen, J. Biomed. Opt. 12, 024020 (2007).

13. B. Varghese, V. Rajan, T. G. Van Leeuwen, and W. Steenbergen, Opt. Express 15(15), 9157-9165 (2007).

14. B. Varghese, V. Rajan, T. G. Van Leeuwen, and W. Steenbergen, (submitted for publication)

15. G. E. Nilsson, Med. Biol. Eng. Comput. 22, 343-348 (1984).

16. D. P. Kernick, J. E. Tooke, and A. C. Shore, Eur. J. Physiol. 437, 624-631 (1999). 